\title{
High-efficiency Bragg Grating Enhanced On-chip Photon-number-resolving Detectors
}

\author{
P. L. Mennea ${ }^{1}$, B. Calkins ${ }^{2}$, B. J. Metcalf ${ }^{3}$, T. Gerrits ${ }^{2}$, A. E. Lita ${ }^{2}$, J. C. Gates ${ }^{1}$, W. S. Kolthammer ${ }^{3}$, \\ J. B. Spring ${ }^{3}$ and P. C. Humphries ${ }^{3}$, N. A. Tomlin², A. E. Fox ${ }^{2}$, A. Lamas Linares ${ }^{2}$, R. P. Mirin ${ }^{2}$, \\ S. W. Nam $^{2}$, I. A. Walmsley ${ }^{3}$, P. G. R. Smith ${ }^{1}$ \\ 1. Optoelectronics Research Centre, University of Southampton, Southampton, UK \\ 2. National Institute of Standards and Technology, Boulder, USA \\ 3. Clarendon Laboratory, University of Oxford, Oxford, UK
}

The recent trend towards integration of quantum optics experiments has produced a demand for on-chip single photon detectors with high quantum efficiencies. In previous work we demonstrated integrated photon number resolving detectors for use at telecommunications wavelengths [1], here we outline developments of this design which have enabled improvements in the quantum efficiency, permitting an on-chip detection efficiency of $92 \%$ to be obtained in the device of Fig. 1.

The detectors considered in this work are composed of a tungsten Transition Edge Sensor (TES) coupled with a single-mode waveguide via the evanescent field. Key features of TES detectors include a negligible dark-count rate and the ability to resolve the absorbed photon number; high efficiencies of up to $98 \%$ have also been achieved with discrete normal-incidence TES detectors [2]. Here the waveguide was patterned via the process of direct UV writing into a photosensitive germanosilica core layer, $5.5 \mu \mathrm{m}$ thick, deposited via Flame Hydrolysis Deposition (FHD). A thermal oxide layer formed the under-cladding, with a silicon substrate. The detector geometry used in these devices was a $10 \mu \mathrm{m} \times 10 \mu \mathrm{m}$ central TES region with a pair of $100 \mu \mathrm{m}$ long absorbers. A gold strip above each of these absorbers was used to aid thermalisation, allowing a detection event to be registered before the thermal energy is lost to the substrate. Three of these detectors were positioned in sequence along the waveguide, as shown in Fig. 1(b), together forming a single detector of higher efficiency. A single in-band Bragg reflector was co-patterned with the waveguide at the end of the device to provide a double-pass increased absorption.

(a)

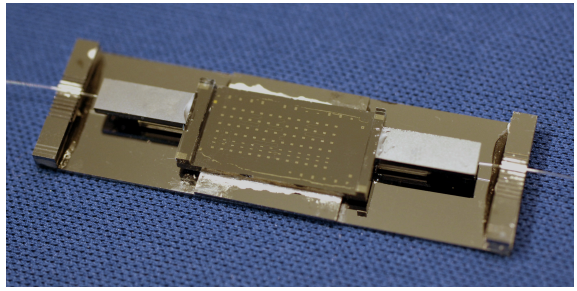

(b)

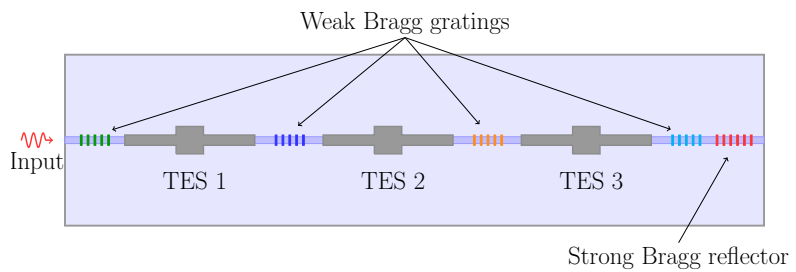

Fig. 1 a) An image of the fabricated high efficiency detector chip, mounted and pigtailed. b) Schematic layout of the high efficiency detector chip.

Both ends of the waveguide were pigtailed with fibre v-grooves, attached using an index matched epoxy. Careful mounting of the chip was required to provide thermal anchoring of the pigtails and substrate. An operating temperature below $100 \mathrm{mK}$ was reached using an adiabatic demagnetization refrigerator, and the efficiency of the three elements characterised using an attenuated $1550 \mathrm{~nm}$ laser source. The average detection efficiency of the three TES detectors was $46.5 \%$ for the optimal TM polarisation, giving a total on-chip detection efficiency of $84.7 \%$, closely matching the modelled value. This was further increased within the narrow reflector bandwidth, for which a second pass occurs, to $92 \%$.

Interrogation of the device was also conducted at room temperature using classical light by means of a set of out-of-band Bragg gratings between each of the detectors, again co-patterned with the waveguide. Measuring the reflection spectrum from both launch directions permitted a relative power profile to be obtained using the method detailed in [3]. By this means the loss of the waveguide and each detector was measured; it was verified that the classical absorption was in good agreement with the measured device quantum efficiency.

We shall present details of the design and fabrication of these devices, as well as highlighting the use of classical light in their characterisation.

\section{References}

[1] T. Gerrits, N. Thomas-Peter, J. C. Gates, A. E. Lita, B. J. Metcalf, B. Calkins, N. A. Tomlin, A. E. Fox, A. Lamas-Linares, J. B. Spring, N. K. Langford, R. P. Mirin, P. G. R. Smith, I. A. Walmsley, and S. W. Nam, "On-chip, photon-number-resolving, telecommunication-band detectors for scalable photonic information processing," Phys. Rev. A 84, 060301 (2011).

[2] D. Fukuda, G. Fujii, T. Numata, K. Amemiya, A. Yoshizawa, H. Tsuchida, H. Fujino, H. Ishii, T. Itatani, S. Inoue, and T.Zama, "Titaniumbased transition-edge photon number resolving detector with $98 \%$ detection efficiency with index-matched small-gap fiber coupling," Opt. Express 19, 870 (2011)

[3] H. L. Rogers, S. Ambran, C. Holmes, P. G. R. Smith, and J. C. Gates, "In situ loss measurement of direct UV-written waveguides using integrated Bragg gratings," Opt. Lett. 35, 28492851 (2010). 